\title{
New Techniques for Radar Coherent Range Ambiguity Resolution
}

\author{
Feng-ling Lin and Michael Steiner \\ Naval Research Laboratory \\ 4555 Overlook Ave., S.W. \\ Washington, D.C. 20375
}

\begin{abstract}
A new method of target coherent range ambiguity resolution is presented which is substantially different from previously proposed methods. The new target ranging eliminates the generation of target ghosts associated with most conventional techniques. A range ambiguous radar with a medium to high pulse repetition frequency is assumed. Clutter is assumed to be present in multiple range intervals. The method utilizes constant frequency pulses that change phase pseudorandomly from pulse to pulse. The phase progression of the received pulses are correlated with a bank of matched filters constructed using phase progressions representative of the different range intervals. Performance degradation of target detection using the pseudo-random phases is also examined. Target detection with strictly pseudo-random phases is found to degrade in comparison to the conventional constant phase methods. A technique that utilizes both constant phase and pseudo-random phases is proposed that performs both detection and target ranging well.
\end{abstract}

\section{INTRODUCTION}

Medium to high pulse repetition frequency (PRF) radars are often employed to increase the unambiguous Doppler blind speed of the radar. The major benefit of using a high PRF is that the clutter is highly correlated and easily canceled, leaving a large percentage of the Doppler space available for target detection. A disadvantage of using high PRF's is that the radar becomes highly range ambiguous. Other disadvantages are the increased receiver dynamic range required to cancel the clutter and the effects of eclipsing due to a relatively high duty cycle.

Target ranging for pulse Doppler range ambiguous radars has traditionally been accomplished by using one of two methods. The first method is to use multiple bursts of pulses so that each successive burst has a different PRF. Targets will appear in different range cells from burst to burst as a function of the PRF and unambiguous range. The Chinese remainder theorem can be used to unravel a single target in a burst. When multiple detections are present in a burst this method may generate ghosts or false targets. This technique has been examined at length, see [1], [2, p.440], [3, p.17.20] and $[4$, p.116].

The other method which has been applied is to linearly frequency modulate the transmitted pulse. First a group of pulses is used without frequency modulation (FM) to determine the true Doppler frequency of the target. Then FM is applied and the difference frequency between the local oscillator and the received signal is measured. By subtracting the measured Doppler, the range can be determined. This method, like the prior one, also is susceptible to ghosting when multiple targets are present in an antenna beam. This technique has also been extensively researched, see [3. p.17.23], [2, p.448] and [5]. Other techniques that could be utilized are pulse width modulation (PWM) and pulse amplitude modulation (PAM). These techniques may degrade due to limiting, eclipsing, and range-gate straddle losses.

The target coherent range ambiguity resolution methodology presented in this paper is substantially different from the previously proposed methods. It is based on constant frequency pulses that are phase modulated pulse-topulse. Phase modulation is a useful feature for distinguishing target range. Two techniques are shown which can unwrap a target in a single range cell, and are not susceptible to ghosts. Ghosts arise from the conventional algorithms discussed above that rely on unwrapping detections from different range cells using different PRFs. However, phase modulation adds some complexity in canceling the clutter from different ambiguous target ranges. Note that previously, it was believed that clutter could not be canceled from multipletime-around echoes with a random phase system [6, p.241]. Carlsson [7] was the first to show that it is possible to cancel the multiple-time-around intervals of clutter by placing the zeroes of an MTI filter weights on the clutter from different intervals. By formulating the problem using optimal matched filter theory, we extend Carlsson's work and show that both clutter cancellation and target integration are possible. The amount of clutter cancellation and target integration depends on, among other factors, the number of pulses, or degrees of freedom, and the number of range ambiguous clutter intervals.

Two techniques for resolving ambiguities are presented. The first technique transmits pulses with pseudo-random phases and performs both target detection and range ambiguity resolution in a single coherent processing interval (CPI). Matched filters are designed for every (quantized) case of Doppler and target range cell. Consequently, it is hardware intensive. The second technique requires an estimate of the target Doppler by first transmitting pulses with constant phase. The Doppler estimate is then used to process pseudo-random phased transmitted pulses for both target detection and range ambiguity resolution.

In Section II the problem is stated and the methods are described in detail. In Section III the performance of the methods for target detection is examined through simulation 
and the performance of the techniques for target range ambiguity resolution is shown. Finally, conclusions are drawn in Section IV.

\section{PROBLEM FORMULATION}

We assume that the received signal $y$ is given by $y=s+c$ $+n$, where $s$ is the target echo, $c$ is the clutter and $n$ is the noise. Also $y, s, c$ and $n$ are $N$-dimensional complex vectors where $N$ is the total number of the transmitted pulses. The vectors are composed of the pulse returns of the in-phase and quadrature components of the received signal for a specific range gate (assumed previously pulse compressed if the system has pulse compression) during a single CPI. During any $\mathrm{CPI}$, the radar is at a fixed operating frequency, but can change frequency between CPIs.

Let $R_{\text {unamb }}=c T / 2$ denote the unambiguous range of the radar, where $T$ is the interpulse period and $c$ is the speed of light. The target, when present, is assumed to be in the $N_{t}^{t h}$ range interval; i.e. lies between $R_{\text {unamb }}\left(N_{t}-1\right)$ and $R_{\text {unamb }} N_{t}$ up to a maximum range of $R_{\text {unamb }} N_{\max } \cdot$ It is further assumed that $N_{t}$ is uniformly distributed in the set $\left\{1,2, \ldots, N_{\max _{t}}\right\}$. Let $\phi_{i}$ be the phase of the $i^{\text {th }}$ transmitted pulse. A constant PRF is assumed throughout the investigation. The target echo $s$ can be described by

$$
\alpha_{S}\left(a_{1}, a_{2} e^{j \theta}, a_{3} e^{j 2 \theta}, \ldots, a_{N} e^{j(N-1) \theta}\right),
$$

where $\alpha_{s}=S e^{j \psi}$ with $\psi$ uniformly distributed in $[0,2 \pi], S$ is the target strength, $a_{i}=e^{i \phi_{i}}$ with $\phi_{i}$ uniformly distributed in $[0,2 \pi]$, and $\theta \in[0,2 \pi]$ is the Doppler shift normalized with respect to the PRF. We denote the normalized Doppler frequency by $f=\theta / 2 \pi$. Assume that the returned target signal $s_{i}$ is $i^{\text {th }}$ time ambiguous, where $i=1, \ldots, N_{\max }$. For example, if $i=3$ we have

$$
s_{3}=\alpha_{s}\left(0,0, a_{1}, a_{2} e^{j \theta}, \ldots, a_{N-2} e^{j(N-3) \theta}\right) .
$$

Note that $s_{i}=s_{i}(f)$ is a function of the normalized Doppler frequency $f$. Consequently, the resultant target echo can be written as $\sum_{i=1}^{N_{\max }} \delta_{N_{t}, i} s_{i}$, where $\delta_{N_{t}, i}$ is one when $i=N_{t}$ and zero otherwise.

The thermal noise $\boldsymbol{n}$ is assumed to be Gaussian-distributed with zero mean and variance $\sigma_{n}^{2}$. The covariance matrix of $\boldsymbol{n}$, defined as $E\left(\boldsymbol{n}^{*} \boldsymbol{n}^{\mathrm{T}}\right)$ where ${ }^{*}$ denotes conjugate and $\boldsymbol{T}$ denotes transpose, is $\sigma_{n}^{2} I_{N}$, where $I_{N}$ is the $N \times N$ identity matrix.

The clutter $c$ is also assumed to be Gaussian-distributed, physically present up to a range of $R_{\text {unamb }} N_{\max c}$, where $N_{\max _{c}}$ is the maximum number of clutter range interval. Let $c s_{i, j}$ denote the $i^{t h}$ sample of the $j^{t h}$ interval clutter. Then $c s_{i, j}$ is related to the $i^{i h}$ element $c_{i}$ of $c$ as follows :

$$
\begin{aligned}
& c_{1}=c s_{1,1}, c_{2}=c s_{2,1}+c s_{1,2}, c_{3}=c s_{3,1}+c s_{2,2}+c s_{1,3}, \ldots, \\
& c_{N}=c s_{N, 1}+c s_{N-1,2}+\ldots+c s_{1, N} .
\end{aligned}
$$

Note that the multiple-time-around clutter progressively builds up as $i$ in $c_{i}$ increases. This is because there cannot be any multiple-time-around clutter in the first pulse. The multiple-time-around clutter from then on includes returns from its range interval and all preceding range intervals. The clutter has a Gaussian-shaped power spectral density

$$
E\left(c s_{i, j}^{*} c s_{i+k, j}\right)=a_{i}^{*} a_{i+k} \sigma_{j}^{2} \rho^{k^{2}}
$$

for the $j^{\text {th }}$-time-around clutter for all $i, k$, where $\sigma_{j}^{2}$ is the variance of the $J^{\text {th }}$-time-around clutter. Here $\rho$ can be determined by $\rho=e^{-2(\pi \sigma T)^{2}}$ where $\sigma T$ is the normalized spectral width of the clutter [2, p.255]. We assume that the clutter from different intervals are uncorrelated, i.e. $E\left(c_{i, j}^{*} c_{i+k, l}\right)=0$ for all $i, k$ when $j \neq l$. Note that we have implicitly assumed that each $j^{\text {th }}$-time-around clutter source has the same spectral density, although such assumptions are not needed in most of the theory presented.

The covariance matrix of the interference or clutter plus noise, $\quad R_{c+n}=E\left(c^{*} c^{T}\right)+\sigma_{n}^{2} I_{N}, \quad$ is assumed known. $R_{c+n}=\left[r_{i, j}\right]$ is found by considering the relationship between $c$ and $c s_{i, j}$ in (1) and by applying (2). This results in

$$
r_{i, j}=\sum_{k=1}^{\min (i, j)} a_{i-k+1}^{*} a_{j-k+1} \sigma_{k}^{2} \rho^{(i-j)^{2}}+\sigma_{n}^{2} \delta_{i, j},
$$

where $\sigma_{k}^{2}$ is the variance of the $k^{\text {th }}$-time-around clutter (if $\sigma_{k}^{2}=0$, then there is no $k^{\text {th }}$-time-around clutter) and $\delta_{i, j}=1$ if $i=j$ and zero otherwise. We now propose two methods valid for both target detection and target ambiguity resolution.

\section{A. Generalized Likelihood Ratio Test}

In the first technique to resolve target range ambiguities, the Generalized Likelihood Ratio Test (GLRT) is employed to perform both target detection and target ambiguity resolution in a single coherent processing interval. Essentially the GLRT consists of a bank of filters: $N_{D P}$ filters covering Doppler and $N_{\max _{t}}$ filters covering the range intervals. Although the GLRT is not necessarily optimal in terms of maximizing the probability of detection for a fixed probability of false alarm, the GLRT tests are often employed due to their ease of implementation and reasonable 
performance (see [8] for a comparison in a radar related case). We consider the case of only one range cell for simplicity, since that is all that is required for target ambiguity resolution with the methods presented. Assume that pulses with pseudo-random phases are transmitted. Let the normalized Doppler space $[0,1]$ be covered by a set of $N_{D P}$ discrete Doppler filters: $\Lambda_{D P}=\left\{\lambda_{1}, \ldots, \lambda_{N_{D P}}\right\}$, where $\lambda_{i}$ 's are the filter center frequencies. To estimate the target range, the GLRT can be derived [9] as

$$
\arg \max _{i} \max _{f \in \Lambda_{D P}} \frac{\left|s_{i}^{\dagger} R_{c+n}^{-1} y\right|}{s_{i}^{T} R_{c+n}^{-1} s_{i}^{*}}
$$

where ${ }^{\dagger}$ denotes transpose conjugate and $R_{c+n}^{-1}$ denotes the inverse of $R_{c+n}$. This technique is hardware intensive since $N_{D P} \times N_{\text {max }}$ matched filters are required for every (quantized) Doppler and target cell.

For detection, the maximum of the filters is compared to a CFAR threshold. Often, the GLRT is normalized so that the filters have the same norm in thermal noise. Furthermore, if the filters have separate cell averaging CFAR's the normalization is irrelevant. Hence for detection, the normalized GLRT (NGLRT) filters are assumed to be $\left|w^{T} y\right| /$ $\|w\|$ where $w=R_{c+n}^{-1} s_{i}$, and $\|w\|$ denotes the Euclidean norm of $w$.

Note that once the NGLRT detection filter outputs are computed, the range can be estimated from Eq. (3). It is found through simulation however, when the clutter is nearly canceled the denominator scale factors give only a small improvement in terms of ambiguity resolution performance.

\section{B. Doppler Estimation Method}

The previous method offers a solution, but is relatively complex to implement. Another method is less complex to implement and also can be used for both target detection and estimating the target location.

This method is called the Doppler Estimation Method (DEM) that consists of two separate steps. The first step is to transmit a batch of $N_{1}$ constant phase pulses. The returns from these pulses are used to determine the target Doppler using a conventional Doppler filter bank (this may be ambiguous depending on the PRF). The technique we utilize to determine the target Doppler is to choose the Doppler corresponding to the Doppler filter that has the maximum output over all filter outputs, for filters designed over different Doppler frequencies. Enough filters are designed so that the Doppler filter separation between the filters is smaller than the theoretical Doppler resolution. The second step is to transmit a batch of $N_{2}$ pseudo-random phase pulses with the same PRF as the first batch. Target detection in the second batch is achieved by using the Doppler estimate found via the first batch of pulses. Target ambiguity resolution for this second batch is accomplished by the NGLRT method that only requires $N_{\max }$ filters. Hence the maximization in Eq. (3), not taking place over all $f$, is accomplished by replacing $s_{i}$ with a corresponding signal with Doppler equal to the estimate. Note that if the target Doppler from the first PRF is known unambiguously, which is often the case with medium to high PRF radars, then the transmitted frequency for the second batch can be made different from that of the first PRF. However, $f$ needs to be renormalized to take this into account. If the target Doppler from the first PRF is not known unambiguously, then the transmitted frequency of the second burst should be the same as the first burst. In the simulations presented in Section III, we assume same transmitted frequency for both batches.

Note that the results of the detection from the second batch could potentially be used for confirmation of the first detection. This has the potential advantage of improved performance over using only a single batch, depending on the detection performance of the second batch. We present simulation results in Section III based on a per batch probability of detection; the results of combining the two batches are dependent on the detection confirmation rule.

\section{PeRformance Results}

In this section we show performance via simulation for the two techniques proposed above. Correlated Gaussiandistributed clutter with spectral width $\sigma T$, thermal noise, and targets are computer generated, based on the models presented in Section II. We assume throughout the simulations that the overall $C N R$ is $30 \mathrm{~dB}, \sigma T$ is 0.0033 and $N_{\max _{1}}$ and $N_{\text {max }_{c}}$ both equal to 15 .

We simulate via Monte Carlo trials a single range cell using 1000 iterations. The threshold for a false alarm rate of 0.001 is first determined using 20,000 iterations. The transmitted phases used in the simulation were chosen randomly from a uniform random number generator, but were fixed in the simulation once chosen. This is necessary due to the number of iterations required to compute the probability of false alarm and the slow speed of inversion of large matrices. However, prior to this, six different sets of phases had been run, and the results differed within $\pm 0.4 \mathrm{~dB}$.

In absence of target, the vector $y$ that contains thermal noise and clutter is generated differently at each Monte Carlo run. For each run, $y$ is a vector from a Gaussian random process with covariance matrix $R_{c+n}$. In order to generate the multiple-time-around clutter, the clutter responses for different intervals are obtained separately followed by adding them together taking into account the phases of the transmitted pulses. The correlated clutter samples, assuming first interval clutter only, can be easily generated by first starting with a Gaussian vector that contains independent components with zero mean and variance equal to one, and multiplying this vector by a matrix $A$, where $\mathrm{A}$ is a lower 
triangular matrix determined by the correlation coefficient $\rho$ and $R=A^{*} A^{T}$. Here $R$ is a clutter covariance matrix designed for a single clutter source with transmitted phases that are the same. Next, the vector is multiplied by a complex constant of unit magnitude and the assumed transmitted phase. This procedure is repeated for the multiple interval clutter sources, with the exception that the multiple interval clutter sources are derived by initially zero-filling the first $i-I$ samples of the $i^{\text {th }}$-time-around clutter and truncating the vector by eliminating the last $\mathrm{N}-\mathrm{i}+1$ samples. Finally, the clutter is summed and thermal noise is added.

Since there are multiple clutter sources, the $C N R$ is the ratio of the sum of the power of all the clutter sources divided by the sum of the noise power. We set the noise power equal to 1. The overall $C N R$ is shown to be

$$
\left(N \sigma_{1}^{2}+(N-1) \sigma_{2}^{2}+\ldots+\left(N-N_{\max _{c}}+1\right) \sigma_{N_{\max _{c}}^{2}}^{2}\right) / N,
$$

where $\sigma_{k}^{2}$ is the clutter power or variance of the $k^{\text {th }}$-timearound clutter. Assuming $\sigma_{k}^{2}$ 's are identical leads to a solution for $\sigma_{k}^{2}$.

In the case where the target is present, the target return is added to the clutter and noise. The target is generated as a function of its unambiguous range. For example, if the first target return were in the 3rd range interval, then $s_{3}=\alpha_{s}\left(0,0, a_{1}, a_{2} e^{j \theta}, \ldots, a_{N-2} e^{j(N-3) \theta}\right)$. The $S N R$ is given by $S N R=\frac{\left|\alpha_{s}\right|^{2}}{N} \sum_{k=1}^{N}\left|s_{j, k}\right|^{2}$, where $s_{j, k}$ is the $k^{\text {th }}$ component of $s_{j}$ ( $j=3$ in the above example). For any fixed $i$ or $s_{i}$, this becomes $S N R=(N-i+1)\left|\alpha_{s}\right|^{2} / N$. We have assumed that the target has unknown cross section. Hence we model the $S N R$ as unknown, but constant, independent of the range interval. Note that if one had a priori knowledge of the target cross section, performance could be improved by modeling the $S N R$ as a function of range interval. In order to assure that each Monte Carlo iteration has the same SNR independent of the target range interval $i, \alpha_{s}$ needs to be a function of $i$. In the following we present the simulated detection and target range ambiguity resolution performance results.

\section{A. Detection Performance}

The use of pseudo-random phase as opposed to the conventional constant phase pulses will result in detection performance degradation. However, it has been found theoretically that optimal filters can be designed with little loss in many cases. Detection performance for the GLRT is obtained to assess the performance degradation as compared with a conventional system.

In Fig. 1 the detection results are shown for the constant phase and the GLRT with pseudo-random phases. In all the curves the total number of pulses on target is 80 . The $S N R$ shown is the input $S N R$, hence it is lower than the output $S N R$. For a perfect integrator, the integration gain would be $19 \mathrm{~dB}$ for 80 pulses. It is preferable for this paper to use the input $S N R$ as a basis for comparing different techniques since it is a fixed quantity, as opposed to the output $S N R$ that can vary for different methods.

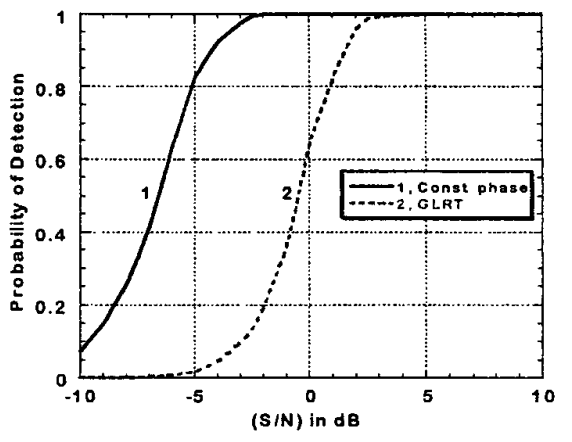

Fig. 1. Detection Performance of the GLRT.

The threshold is set so that the probability of false alarm is 0.001 , a relatively high false alarm rate is necessary for simulation purposes. There are 65 Doppler filters uniformly spaced between 0.1 and 0.9 having an increment of 0.0125 : $\Lambda_{D P}=\{0.1,0.0125, \ldots, 0.9\}$. Note that we begin filtering at $f$ $=0.1$ and end at $f=0.9$ to avoid clutter contamination, while preserving $80 \%$ of the Doppler region. The normalized Doppler of the target is assumed to be $f=0.33125$ which lies halfway between the $\Lambda_{D P}$ discrete levels. The actual target interval $N_{t}$ is uniformly distributed in the set $\left\{1,2, \ldots, N_{\max _{t}}\right\}$.

Curve 2 (dashed line) in Fig. 1 shows the performance of the GLRT. Note that $P_{d}=0.5$ occurs at slightly less than $0 \mathrm{~dB}$, which is fairly good for many targets of interest that are at or above thermal noise. For comparison, the performance of a conventional system is shown in Curve 1 (solid line). The performance of the constant-phase system in absence of clutter case is also obtained but not shown here because it almost coincides with the curve with clutter. The proximity of the two curves illustrates that the filters are effectively cancelling the clutter. For the conventional system, detection is accomplished by using 65 matched filters matched to $\Lambda_{D F}$. This detector compares $\max _{\lambda \in \Lambda_{D P}}\left|s(\lambda)^{\dagger} R_{c+n}^{-1} y\right|$ to a threshold, where $s(\lambda)$ represents a signal with normalized Doppler frequency $f=\lambda$. Note that $R_{c+n}$ and $s(\lambda)$ are computed based on 65 pulses, since the first 15 fill pulses are used so that the clutter will be in steady state prior to detection. Comparison between Cuve 1 and Curve 2 shows that there is a $6 \mathrm{~dB}$ loss in the detection performance in using the pseudo-random phases as opposed to using constant phases. Also the conventional system is less complex to implement. However, as we will see in Section III- $B$, the benefit of using the pseudo-random 
phases is that the target range ambiguity resolution performance is greatly enhanced.

The DEM is also simulated. In this method we divide the 80 pulses into two sets of 40 pulses so that the first 40 pulses have constant phase with the first 15 used as fill pulses, and the remaining 40 pulses have pseudo-random phases. The Doppler filter spacing $\Lambda_{D P}$ for the first set of pulses is the same as previously discussed with 65 filters uniformly spaced in the normalized Doppler space between 0.1 and 0.9 . The target Doppler is estimated as the filter center frequency corresponding to the maximum of the 65 Doppler filter responses. Although better resolution could be obtained through other methods such as interpolation, this technique is sufficient for our purposes. The second set of pulses with pseudo-random phases is also used to estimate the target Doppler. The results are shown in Curves 1 and 4 of Fig. 2. Note that it appears that the first batch of 40 constant phase pulses dominates the performance of the second batch. The added benefit of using the second batch of pulses for detection would be to provide a detection confirmation of the first detection. However, due to the difference in performance, these curves show that there appears to be relatively small benefit in using the remaining 40 pseudorandom pulses to provide improved detection confirmation. These curves assume the target Doppler $f=0.33125$ is in a Doppler filter passband. Curve 1 of Fig. 2 and Curve 1 of Fig. 1 show a loss of about $2 \mathrm{~dB}$ in the detection performance when a conventional constant phase system with 40 pulses is compared with 80 pulses. Also shown in Curves 2 and 3 are the performance when batch 1 is 30 pulses and batch 2 is 50 pulses. Note that the curves separate from Curves 1 and 4 toward each other as consideration of the number of pulses would suggest.

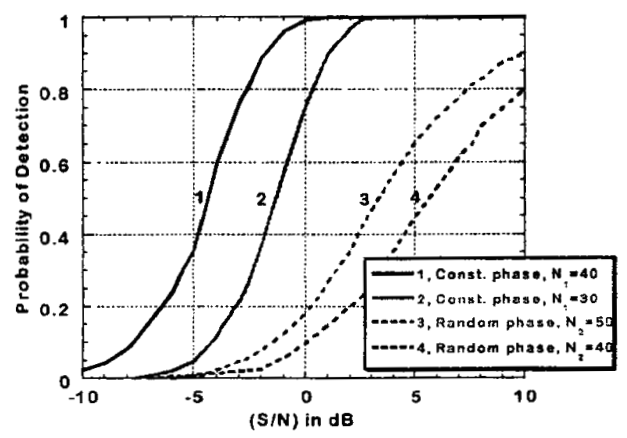

Fig. 2. Detection Perfornance of DEM

\section{B. Target Range Ambiguity Resolution Performance}

In this section we show that the target location ambiguity can be accurately resolved in a single CPI in medium to high PRF radars (it also can be extracted in low PRF radars if desired). All the parameters, such as $C N R, \sigma T$, are the same as before.
In the simulation we chose the actual target interval $N_{t}$ randomly in the set $\left\{1,2, \ldots, N_{\max _{f}}\right\}$. We characterize the performance by the probability of error which is found by the number of times the estimated target interval does not match $N_{t}$ divided by the total number of Monte-Carlo runs, which is 1000. Unlike the previous section where a large number of Monte-Carlo iterations are necessary in order to determine the threshold for a given probability of false alarm, only 1000 iterations are needed here. Hence, we use a different set of randomly chosen transmitted phases and invert a matrix for each iteration. In the simulation, we do not require a detection to be made in the first batch of pulses. This allows performance at low $S N R$ to be evaluated. If only detections are passed assuming a low false alarm rate, the SNRs of the passed detections would typically be high.

The Curve 5 in Fig. 3 shows the performance of the GLRT for a conventional constant phase system. Note, in contrast to the detection curves of Section III-A, this poor performance indicates that constant phases cannot be used to adequately estimate the target range. In Curves 2 thru 4 we show the performance of the GLRT for a pseudo-random phase system as a function of the number of Doppler filters $N_{D P}$ for any fixed target location hypothesis. As in the prior section, the Doppler filters are uniformly spaced in the normalized Doppler space between 0.1 and 0.9 . The performance when $N_{D P} \geq 65$ is very good considering that there are 15 intervals of range ambiguous clutter $\left(N_{\operatorname{rax}_{c}}=15\right)$. Note that there is a threshold where the performance drops off dramatically. When only 33 Doppler filters are used, the performance is poor compared with 65 or 129 Doppler filters. However, for 129 Doppler filters, the total number of filters required in Eq. (3) is $1935(129 \times 15)$ which is rather substantial. In Curve 1 we also show the performance of the GLRT when the Doppler is known so that the maximization in Eq. (3) is only over a set of $N_{\max }$ filters.

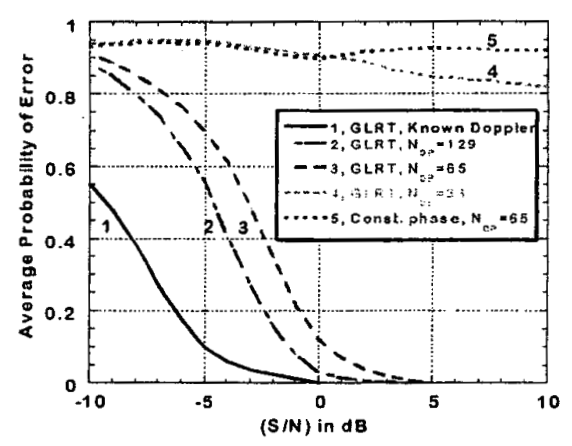

Fig. 3. Estimation Performance of the GLRT.

In Fig. 4 we show the performance of the DEM. In Curve 5, $N_{I}=40, N_{2}=40$ (denoted by Case 1), $N_{D P}=65$. An estimate of the Doppler is made using the first 40 constant 
phase pulses that is subsequently used in the remaining 40 pseudo-random phase pulses to form an estimate of the target interval. The performance is very good and comparable to the performance of the GLRT which utilizes 80 pulses. The GLRT with $N_{D P}=65$ requires 975 filters for target ambiguity resolution, as compared with 80 for the DEM $\langle 65$ filters for the first step of constant phase Doppler estimation, and 15 for the second step of estimating the target interval with the pseudo-random phases) - a substantial savings in complexity.

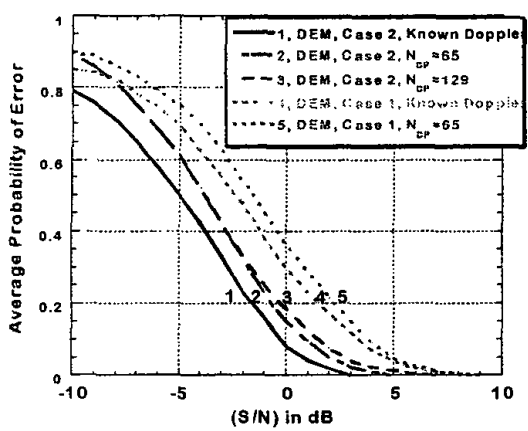

Fig. 4. Estimation Performance of DEM.

In Curves 2 and 3, we show the performance when $N_{l}=$ $30, N_{2}=50$ (denoted by Case 2) for $N_{D P}=65$ and $N_{D P}=$ 129 , respectively. Note that although the number of pulses in the two batches is different $\left(N_{l} \neq N_{2}\right)$, better performance results for this asymmetric case than when $N_{1}=N_{2}$.

In Curves 1 and 4 the performance is shown when the Doppler is known and the target location is estimated in the second batch of pulses. In Curve $1, N_{2}=50$, and in Curve 4, $N_{2}=40$. Note that there is only a $1 \mathrm{~dB}$ loss between the cases when the Doppler is known and estimated via the first batch of pulses.

\section{CONCLUSIONS}

Conventional techniques for target ranging for pulse Doppler range ambiguous radars involving multiple coherent bursts, each burst with a different PRF, to unwrap the target position. These techniques are susceptible to ghosting because detections take place in different range cells from one coherent burst to another. The new techniques proposed here do not result in these types of ghosts, since the target ambiguity resolution is accomplished on a single range cell basis. The target position can be estimated unambiguously in clutter with a single CPI when pulses with pseudo-random phases are transmitted. These phase sequences were found to be an excellent discriminant in terms of unambiguously estimating the target location.

The DEM method limits target detection loss to a few $\mathrm{dB}$ compared with conventional constant phase systems. This is primarily due to the strong detection performance of the first constant phase batch of pulses used in the DEM. However, when strictly pseudo-random phases are relied upon for detection as in the GLRT, detection losses were found to be larger in clutter as compared with the conventional systems. It is believed that for many stronger targets of interest, and in environments where clutter does not overly restrict performance, detection with pseudo-random phases can be useful.

Detection loss can be explained heuristically as follows. In order to cancel the clutter from different intervals, degrees of freedom of the Doppler filter weights are used to essentially place zeroes at the locations of the multiple-time-around clutter. Placing these zeroes results in loss of integration gain, since the weights are different from what is required for an ideal integrator. The more the multiple-time-around clutter is cancelled, the larger the integration loss. However if all the multiple-time-around clutter is cancelled, then the most this loss should be is the theoretical integration gain in thermal noise. From Fig. 1, the $S N R$ loss is approximately $6 \mathrm{~dB}$ when comparing the conventional constant phase with the GLRT with pseudo-random phases. This is much less than the theoretical integration loss of $10 \log 80 \cong 19 \mathrm{~dB}$, indicating there are still enough degrees of freedom remaining for integration gain.

Two methods were proposed to accomplish target ambiguity resolution. The GLRT provides the best ambiguity resolution performance but suffers in terms of complexity and detection performance. The DEM provides a practical approach of reasonable complexity and good detection and ambiguity resolution performance.

Note that in these simulations we assume 15 intervals of range ambiguous clutter. In practice, only a few intervals may be present. Both detection and range ambiguity resolution performance generally improves when fewer ambiguous intervals of clutter are present.

\section{REFERENCES}

[1] W. Skillman and D. Mooney, "Multiple high-prf ranging," Proc. of IRE $5^{\text {th }}$ National Convention Military Electronics, pp.37-40, 1961.

[2] D. Curtis Schleher, MTI and Pulse Doppler Radar, Norwood, MA: Artech House, 1991.

[3] M. 1. Skolnik, Radar Handbook, $2^{\text {ad }}$ ed., New York, NY: McGraw-Hill, 1990.

[4] S. A. Hovanessian, Radar System Design and Analysis, Norwood, MA: Artech House, 1984.

[5] G. Hetrich, "Frequency modulation techniques as applied to puise Doppler radar," JRE Conv. Rec., vol. 5, 1962.

[6] D. K. Barton, Modern Radar System Analysis, Norwood, MA: Artech House, 1988.

[7] S. Carlsson, "MTl-filtering for multiple time around clutter suppression in coherent on receive radars," Advances in Radar Techniques (J. Clark, ed.), pp.354-357, London, UK: Peter Peregrinus, 1985.

[8] L. E. Brennan, I. S. Reed, and W. Sollfrey, "A comparison of averagelikelihood and maximum-likelihood ratio tests for detecting radar targets of unknown Doppler frequency," IEEE Trans. Inform. Theory, vol. 14, pp.104-110, January 1968.

[9] L. E. Brennan and 1. S. Reed, "Optimum processing of unequally spaced radar pulse trains for clutter rejection," IEEE Trans. Aerospace and Electronic Systems, vol. 4, pp.474-477, May 1968. 